\title{
RESIDUAL STRESS ANALYSIS IN AA7449 AS-QUENCHED THICK PLATES USING NEUTRONS AND FE MODELLING
}

\author{
N. Chobaut ${ }^{1}$, J. Repper ${ }^{2}$, T. Pirling ${ }^{3}$, D. Carron ${ }^{4}$ and J-M. Drezet ${ }^{1}$ \\ ${ }^{1}$ Ecole Polytechnique Fédérale de Lausanne, Laboratoire de Simulation des Matériaux, station 12, 1015 Lausanne, \\ Switzerland \\ ${ }^{2}$ Paul Scherrer Institut, Materials Science and Simulation, ASQ/NUM, 5232 Villigen PSI, Switzerland \\ ${ }^{3}$ Institut Laue-Langevin, 6, rue Jules Horowitz, 38042 Grenoble, France \\ ${ }^{4}$ LIMATB, Université de Bretagne-Sud / UEB, Centre de Recherche Ch. Huygens, Rue de Saint Maudé, BP 92116, \\ 56321 Lorient Cedex, France
}

Keywords: Aluminium alloy, residual stress, neutron diffraction, precipitation, modelling

\begin{abstract}
In the current trend toward thicker aluminium plates, a major concern is the generation of high internal stresses during quenching, which can cause distortions during machining and pose serious safety concerns. Although the material is stretched after quench, substantially reducing residual stresses, they are not fully suppressed. In addition, the cooling rate is not large enough at the core of such thick plates to prevent any precipitation. This has a great impact on the efficiency of ageing. In this work, residual stress distributions in a heat-treatable aluminium alloy AA7449 thick plate in the as-quenched state measured by neutron diffraction are presented. A comparison between single (311) diffraction peak and multiple peaks analysis using Pawley algorithm is shown. The variation of the stress free reference value through the plate thickness is discussed and measured stresses are compared with residual stresses predicted by a thermomechanical finite element model of quenching.
\end{abstract}

\section{Introduction}

In the processing route of heat-treatable aluminium alloys (AA), a critical step is quenching from the solutionising temperature. High cooling rates are desired to avoid the formation of coarse precipitates that would reduce mechanical properties. Fast quenching, however, cannot be achieved in the center of thick components, where the quenching rate can be more than one order of magnitude lower than that at the surface. The existence of a thermal gradient between core and surface leads to differential thermal deformation which is accommodated by elastic and plastic deformation to satisfy the compatibility of deformation. Whenever the material yields, residual stress (RS) remains after quenching due to the irreversibility of plastic deformation. The final stress pattern of biaxial compression at the skin balanced by triaxial tension at the core is referred to as the "skin-core" effect [1]. After quenching, the RS state may cause both unacceptable distortions during machining and a dramatically reduced service life time. Since RS relaxation is low during ageing treatments, thick components are usually stress relieved prior to ageing. This is achieved by applying plastic deformation: stretching from 1.5 to $3 \%$ in the rolling direction (L) can reduce RS by a factor of approximately 10 [2]. However, complex geometries or thicker components like thick sheets require other approaches. Reducing the quenching rate by using boiling water for instance leads to lower RS [3]. For high quench 
sensitive alloys, however, slow quenching has the drawback to decrease the hardening potential. As a consequence, the ideal quench of heat treatable AA is always a compromise between final properties and RS.

Many researchers have built thermo-mechanical (TM) models to predict RS build-up in thick AA components [3-5]. To validate and improve the numerical models, results were compared with experimental measurements of RS using a wide range of methods such as hole drilling, layer removal, crack compliance, ultrasounds and diffraction. While the agreement between experiments and simulations is good for relatively thin components, thick components raise serious challenges. Firstly, for a reliable prediction of RS the numerical model must take into account metallurgical aspects since the yield strength depends on the precipitation state [5]. Secondly, the experimental method must be able to determine RS deep inside the bulk. For thick components long penetration paths are required. Neutron diffraction (ND) has proved to be a suitable method for aluminium components, as aluminium is rather transparent to neutrons [1].

This paper presents the ND stress measurements of as-quenched AA7449 plates carried out at two independent facilities. The comparison with the predictions of a simple TM model highlights the need for a thermo-metallurgical-mechanical (TMM) model that takes into account precipitation during quenching.

\section{Sample}

The as-quenched AA7449 $75 \mathrm{~mm}$ thick plate was received from Constellium CRV. Preliminary finite element (FE) calculations were performed to determine the plate dimensions in order to avoid edge effects on RS at the plate center. Figure 1 shows the plate geometry and the simplifying equations, assuming an infinite plate in the $\mathrm{x}$ and $\mathrm{y}$ (in-plane) directions. With this assumption, plane-stress conditions are met within the plate.

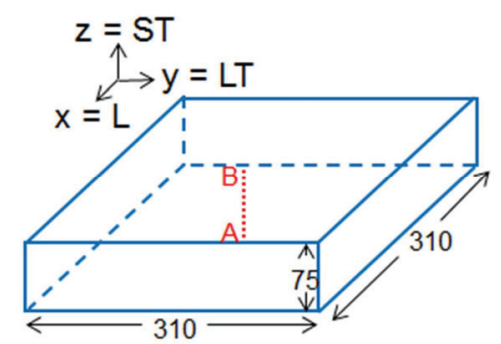

$$
\begin{gathered}
\sigma_{x x}=\sigma_{y y}: \text { large plate without texture } \\
\sigma_{x y}=\sigma_{x z}=\sigma_{y z}=0: \text { no shear } \\
\underline{\operatorname{div}}(\underline{\underline{\sigma}})=\underline{0} \Rightarrow \frac{\partial \sigma_{z z}}{\partial z}=0 ; \sigma_{z z}( \pm h)=0 \Rightarrow \sigma_{z z}(z)=0
\end{gathered}
$$

Figure 1. Sketch of the AA7449 plate geometry. RS were measured by ND at the center of the plate through the thickness from A to B (left). The measurement directions relative to the rolling direction are longitudinal (L), long transverse (LT) and short transverse (ST). $h$ is the half-thickness of the sample. The equation given on the right hand side are valid along $[\mathrm{AB}]$ (right).

FE calculations have shown that equations [1.1] to [1.3] are verified along the through-thickness segment $[\mathrm{AB}]$ of the $310 \mathrm{~mm}$ (L) x $310 \mathrm{~mm}$ (LT) x $75 \mathrm{~mm}$ (ST) plate. As chemical inhomogeneities have been reported by Godard through the thickness of thick aluminium hot rolled plates [5], a reference comb specimen was machined out from the plate using electrodischarge machining (EDM), which is known for its little influence depth on the material $(<0.1 \mathrm{~mm})$. This specimen was used to measure the variation of the stress-free reference value, $d_{0}$, through the plate thickness. 
$\underline{\mathrm{RS} \text { analysis using ND }}$

\section{Experimental methods and modelling}

Strains are obtained by measuring with high accuracy the interplanar distance which acts as an internal strain gauge. In the $\mathrm{x}$ direction, the $\varepsilon_{x x}$ component of the elastic strain tensor, $\underline{\underline{\varepsilon}}$, is derived from the interplanar distance, $d_{x}$, as follows:

$$
\varepsilon_{x x}=\frac{d_{x}-d_{0}}{d_{0}}
$$

Stresses are then calculated using the generalised Hooke's law, which writes using Einstein summation convention:

$$
\sigma_{i j}=\frac{E}{1+v} \varepsilon_{i j}+\frac{E v}{(1+v)(1-2 v)}(\operatorname{tr} \underline{\underline{\varepsilon}}) \delta_{i j}
$$

where $E$ the elastic modulus (71 MPa) and $v$ the Poisson's ratio (0.3). Assuming plane stress conditions leads to:

$$
d_{0}=\frac{2 v}{(1+v)} d_{x}+\frac{(1-v)}{(1+v)} d_{z}
$$

where $d_{x}$ and $d_{z}$ are the interplanar distance in the longitudinal (x) and short transverse (z) directions. This relationship will be compared with $d_{0}$ measurements using the EDM sample.

Monochromatic ND on SALSA. Residual strains were measured at SALSA located at the international Institut Laue-Langevin (ILL) in Grenoble, France. Due to the monochromatic character of SALSA (wavelength is fixed and diffraction angle is measured), only one diffraction peak is recorded, usually the (311) reflection since it exhibits low intergranular strains.

Time-of-flight ND on POLDI. Residual strains were measured by means of the time-of-flight neutron diffractometer POLDI (Pulse-OverLap Diffractometer) located at the spallation source SINQ at the Paul Scherrer Institut (PSI) in Villigen, Switzerland. Due to its time-of-flight character (diffraction angle is fixed and wavelength is determined) POLDI records multiple

Bragg reflections at the same time. This gives insight into texture and enables the calculation of an average lattice parameter using a full diffraction pattern Pawley analysis [6], thus reducing the effects of anisotropy.

\section{Modelling}

The quenching in cold water of an AA7449 aluminium thick plate was simulated using a 2D plane-stress TM model, similarly to Jeanmart and Bouvaist [3]. A temperature dependent heat transfer coefficient was provided by Constellium and the TM model was built in Abaqus 6.10. The aluminium alloy was modelled as an elasto-viscoplastic (EVP) material. Thermomechanical testing on a Gleeble ${ }^{\circledR} 3500$ machine using interrupted quenching tests was performed at Université de Bretagne Sud (UBS) in Lorient, France. Preliminary tensile tests at temperatures of $472^{\circ} \mathrm{C}$ (no quenching), $400^{\circ} \mathrm{C}, 350^{\circ} \mathrm{C}, 250^{\circ} \mathrm{C}$ and $20^{\circ} \mathrm{C}$ using an axial extensometer were carried out to determine the input parameters of the constitutive mechanical model using the identification methodology described in the work of Carron et al. [7]. Further testings are currently performed to optimise the specimen geometry according to the specific thermal and mechanical treatment conditions required. 


\section{Evidence of texture}

\section{Results and Discussion}

Figure 2 shows the two strongest peaks corresponding to the (311) and (220) diffracting planes measured at POLDI using a gauge volume $(\mathrm{GV})$ of $3.8 \times 6 \times 1.5 \mathrm{~mm}^{3}$. Measurements are shown at the mid-thickness $(z=0)$ and at the surface of both the $d_{0}$ reference specimen and the plate.
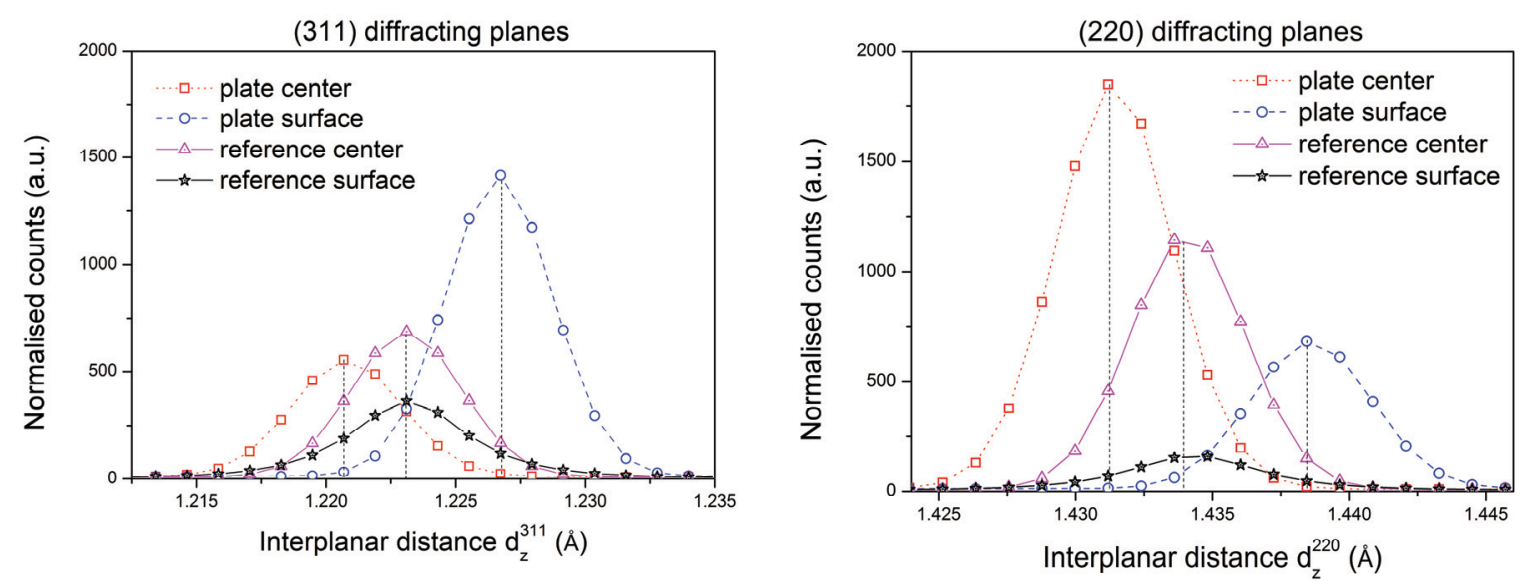

Figure 2. Diffraction peaks (individual fit) measured at the mid-thickness $(z=0)$ and surface of the plate and the reference specimen (POLDI diffractometer). Left: (311) diffracting planes; right: (220) diffracting planes.

Figure 2 illustrates the peak shift toward lower (resp. higher) interplanar distance for the plate center in tension (resp. for the plate surface in compression). The ratio of the integrated normalised intensities, $r=I^{311} / I^{220}$, gives insight into texture: it is higher at the surface $(r=1.9)$ than at the center $(r=0.3)$. The $d_{0}$ reference specimen exhibits the same trend with a ratio of 2.3 at the surface and 0.6 at the center. In the $75 \mathrm{~mm}$ thick plate, $I^{311}$ is still high enough at the center for good statistics at SALSA and POLDI. If the plate were thicker, either (311) peak would disappear or more counts should be needed for good statistics. However, neutron flux was high enough at SALSA and POLDI to alleviate this texture issue in the $75 \mathrm{~mm}$ thick plate.

\section{$\underline{\text { RS analysis }}$}

Interplanar distance measurements were achieved at SALSA in three perpendicular directions (L, LT and ST) every $5 \mathrm{~mm}$ through the plate thickness from A to B (Figure 1). At POLDI, the interplanar distance was measured in two perpendicular directions (LT and ST). The measurements were conducted at both facilities after ca. 2 month natural ageing. The results obtained at POLDI using both single and multiple peaks (Pawley) analysis using 11 peaks are given in Figure 3 . The stress free reference values $d_{0}$ were determined at the EDM specimen. 

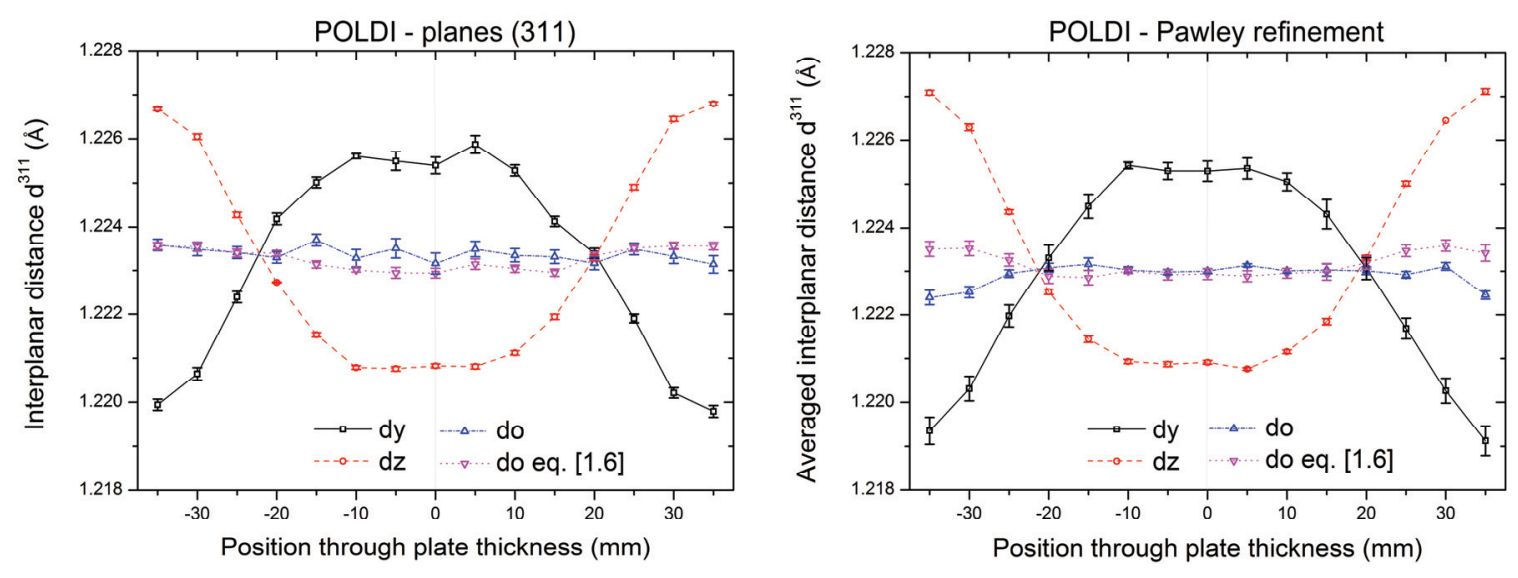

Figure 3. Interplanar distance measurements at POLDI using single peak analysis ((311) planes, left) and Pawley refinement (right).

The data analysed using the (311) reflection is compared to the results of a multiple peak Pawley refinement in figure 3. In both cases, the distribution of the different interplanar distances through the plate thickness is very similar and $d_{0}$ is fairly constant compared to $d_{y}$. Unlike Robinson et al. [4] who reported a decrease in stress free lattice parameter from the surface to the core of AA7075 thick forgings attributed to solute loss, no such variation is observed in the present work. 7449 and 7075 alloys being both quench sensitive, the difference is thought to originate from the different thickness of the components: $75 \mathrm{~mm}$ in the present case and $215 \mathrm{~mm}$ in [4]. The shallow difference between measured and calculated $d_{0}$ is an experimental verification of equation [1.6] which means that no reference specimen is needed in the case of large plates where edge effects are negligible. The calculated reference value was taken for the computation of strains.

In Figure 4, the stress results from SALSA using a GV of $2 \times 2 \times 15 \mathrm{~mm}^{3}$ are compared with those from POLDI determined with the (311) reflection as well as with a multiple peak Pawley analysis.
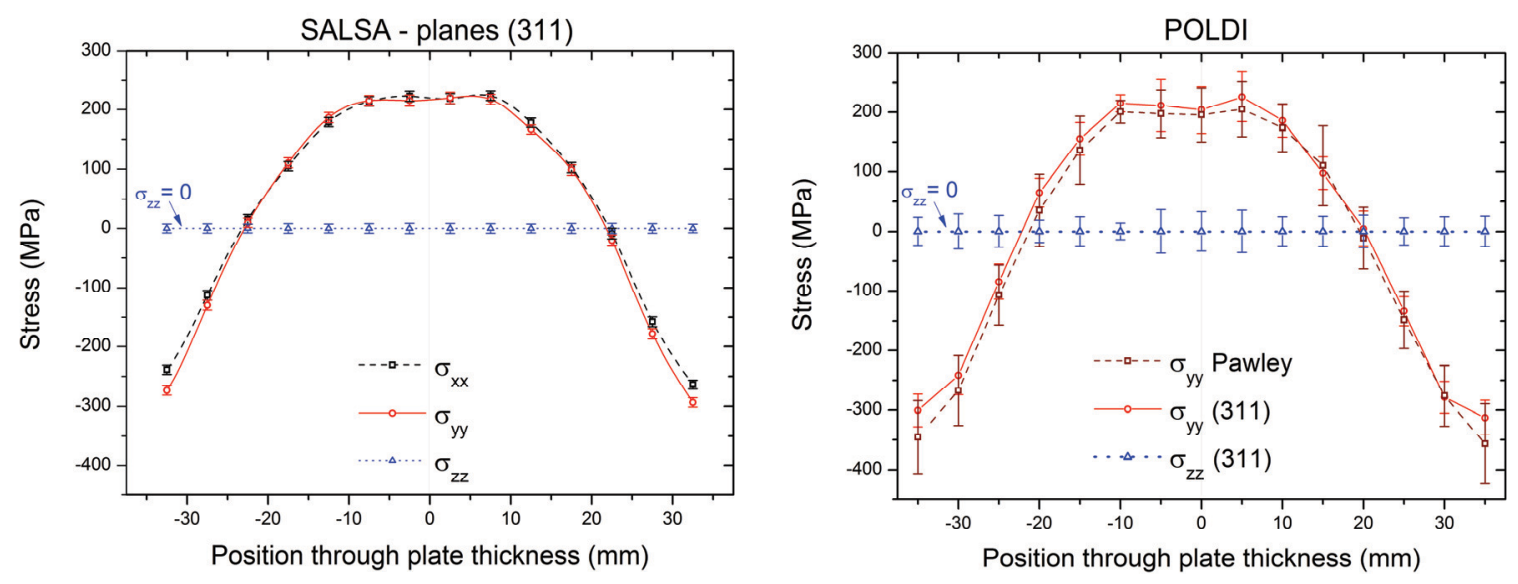

Figure 4. RS profile through the thickness of $75 \mathrm{~mm}$ thick AA7449 plates measured at SALSA (left) and POLDI (right). 
Equation [1.1] is clearly verified at SALSA $\left(\sigma_{x x}=\sigma_{y y}\right)$, justifying why the interplanar distance measurements were measured in only two directions at POLDI. In the three cases (SALSA (311), POLDI (311) and POLDI Pawley), the skin-core effect is verified with about $300 \mathrm{MPa}$ compressive stress at the surface and $200 \mathrm{MPa}$ tensile stress at the center. Stresses are larger in absolute values at the surface than in the center since the quenching rate is higher at the surface than in the center $[8,9]$. SALSA yields RS about $10 \mathrm{MPa}$ higher than those measured at POLDI which are almost identical whatever the method used. The difference in RS between single (311) diffraction peak and multiple peaks analysis is within the experimental uncertainty. This is in agreement with other studies [4]. The major difference between the three cases is the experimental uncertainty; it is around $10 \mathrm{MPa}$ at SALSA, $30 \mathrm{MPa}$ at POLDI using one peak and $50 \mathrm{MPa}$ using Pawley analysis. Further measurements on the less quench sensitive 7040 alloy are planned to determine the effects of quench sensitivity on RS magnitudes. Since the measurements at POLDI and SALSA compare favourably, the results can be used to validate the TM model.

\section{Modelling}

Figure 5 shows the results of the simulation of cold water quenching of the $75 \mathrm{~mm}$ thick AA7449 plate.

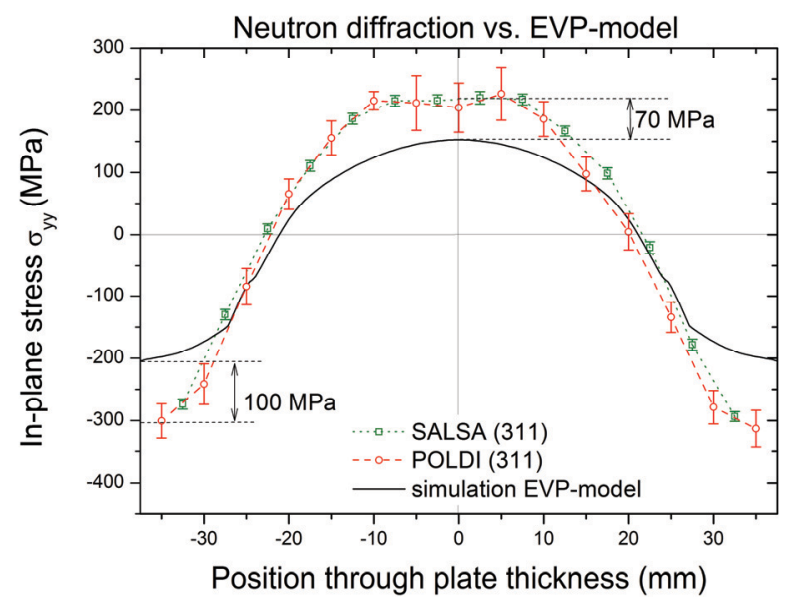

Figure 5. Distribution of as-quenched stresses in $75 \mathrm{~mm}$ thick AA7449 plate: comparison between calculated and measured stresses.

The stress profile determined by FE simulation has the same shape but different magnitude compared to the experimental one. Stresses determined numerically are systematically lower (70 MPa lower at the center) in absolute value than those determined experimentally. Although the mechanical behaviour taken for the simulation is questionable due to the use of an axial extensometer ill-adapted because of the existing axial thermal gradient in the Gleeble specimen, the difference of ca. $100 \mathrm{MPa}$ at the surface is thought to originate from homogeneous precipitation. Hardening precipitates form already during quenching, thus increasing the flow stress and hence decreasing plastic deformation and as a result increasing RS. The numerical 
model definitely needs to be improved by taking into account both homogeneous and heterogeneous precipitation during quenching.

\section{Conclusions}

The measurements of RS in thick heat treatable aluminium plates were presented in this study, using two different diffractometers. SALSA is favoured in terms of measurement time and its high neutron flux. Due to its monochromatic character, however, it may be limited for highly textured materials that are best investigated by time-of-flight instruments like POLDI which collect many diffraction peaks. In the present case of thick hot rolled plates, although some texture was evidenced, data statistics was sufficiently good to carry out single peak analysis on individual Bragg reflections also for POLDI. The agreement between results from the two diffractometers using different analysis procedures is excellent. However, these experimental results compare poorly with the numerical results, not only due to the use of an axial extensometer for strain measurement in the Gleeble machine, but also due to the absence of metallurgical model in the simulation. This work demonstrates that a TMM model is needed to improve the prediction of RS influenced by precipitation in heat-treatable AA.

\section{Acknowledgement}

The authors are grateful to the Swiss Spallation Neutron Source at PSI and the international neutron source at ILL for the provision of beam time. This work is funded by the Competence Center for Materials Science and Technology (http://www.ccmx.ch/) in the frame of the project entitled "Measurements and modelling of residual stress during quenching of thick heat treatable aluminium components in relation to their microstructure" involving EPF Lausanne, PSI Villigen, Univ. de Bretagne Sud Lorient, Constellium CRV and ABB Turbocharger. The Gleeble 3500 machine at Université de Bretagne Sud was co-financed by the European Regional Development Fund (ERDF).

\section{References}

1. Drezet J.-M., et al., Jim Evans Honorary Symposium, Eds. B.-Q. Li, B.-G. Thomas, L. Zhang, F.-M. Doyle and A.-P. Campbell, TMS, 2010: p. 43-52.

2. Muammer, K., J. Culp, and A. Taylan, Journal of Materials Processing Technology, 2006. 174: p. 342-354.

3. Jeanmart, P. and J. Bouvaist, Materials Science and Technology, 1985. 1(10): p. 765-769.

4. $\quad$ Robinson, J.S., et al., Materials Characterization, 2012. 65: p. 73-85.

5. Godard, D., PhD Thesis Institut National Polytechnique de Lorraine (INPL) France. 1999.

6. $\quad$ Pawley, G.S., Journal of Applied Crystallography, 1981. 14: p. 357-361.

7. $\quad$ Carron D., et al., Revue de métallurgie, 2010. 107(10-11): p. 445-448.

8. Li, P., et al., Metallurgical and Materials Transactions B, 2007. 38(4): p. 505-515.

9. Tanner, D.A. and J.S. Robinson, Journal of Materials Processing Technology, 2004. 153154: p. 998-1004. 